\title{
The Planck Length and the Constancy of the Speed of Light in Five Dimensional Spacetime Parametrized with Two Time Coordinates
}

\author{
Christoph Köhn \\ National Space Institute (DTU Space), Technical University of Denmark, Kgs Lyngby, Denmark \\ Email: koehn@space.dtu.dk
}

How to cite this paper: Köhn, C. (2017) The Planck Length and the Constancy of the Speed of Light in Five Dimensional Spacetime Parametrized with Two Time Coordinates. Journal of High Energy Physics, Gravitation and Cosmology, 3, 635-650. https://doi.org/10.4236/jhepgc.2017.34048

Received: August 27, 2017

Accepted: October 9, 2017

Published: October 12, 2017

Copyright $\odot 2017$ by author and Scientific Research Publishing Inc. This work is licensed under the Creative Commons Attribution International License (CC BY 4.0).

http://creativecommons.org/licenses/by/4.0/ (c) (i) Open Access

\begin{abstract}
In relativity and quantum field theory, the vacuum speed of light is assumed to be constant; the range of validity of general relativity is determined by the Planck length. However, there has been no convincing theory explaining the constancy of the light speed. In this paper, we assume a five dimensional spacetime with three spatial dimensions and two local time coordinates giving us a hint about the constancy of the speed of light. By decomposing the five dimensional spacetime vector into four-dimensional vectors for each time dimension and by minimizing the resulting action, for a certain class of additional time dimensions, we observe the existence of a minimal length scale, which we identify as the Planck scale. We derive an expression for the speed of light as a function of space and time and observe the constancy of the vacuum speed of light in the observable universe.
\end{abstract}

\section{Keywords}

Two Time Dimensions, Planck Length, Constancy of the Speed of Light

\section{Introduction}

Since Maxwell's theory of classical electrodynamics [1], it has been known that all electromagnetic waves travel with the speed of light. In 1864 and 1881, experiments performed by Michelson and Morley [2] [3] gave a hint that electromagnetic waves travel equally fast in all inertial systems. This result was confirmed by many more experiments [4] [5] [6] [7] [8] proofing the constancy of the speed of light within the validity of the laboratory setups.

When Albert Einstein derived special relativity [9], he postulated that the speed of light be constant, and he used this assumption as a key ingredient for special relativity. Consequently, he derived that the speed of light is the upper 
velocity limit, whether it be for particles or for information. As such, the limitation of velocities ensures causality; vice versa, information sent with velocities above the speed of light could eventually harm causality.

However, up to now, there have not been sufficiently convincing explanations why the speed of light is constant and why it has the value which it has. Loop quantum gravity, for example, dictates that the velocity of a photon is not defined to be constant, but has a value depending on its frequency [10].

Indeed, there have been suggestions that its value might vary with the age of the universe and that it might not have been constant in the early stages of the universe. Albrecht and Magueijo [11] show that the cosmological evolution equations together with a variable speed of light might solve the horizon, flatness and cosmological constant problem and together with cosmological perturbations the homogeneity and isotropy problem. Deriglazov and Ramírez [12] [13] [14] observed a discrepancy between the speed of light and the critical speed in the theories of spinning particles on curved and electromagnetic backgrounds. Additionally, they noticed that the constancy of the speed of light is closely related with the self-consistent definition of the three-acceleration in general relativity.

The necessity of deriving a theory for quantum gravity resides from the problem that general relativity loses its validity at small length scales [15] [16] and that, for example, quantum electrodynamics stops being a self-consistent theory if gravitational effects [17] are added to the theory. The length scale of these effects is in the order of approximately $10^{-35} \mathrm{~m}$ and was already introduced by Planck in 1899 [18] after the discovery of the Planck's constant when he realized that he could derive a unit system depending on the gravitational constant, speed of light, Planck's constant, Boltzmann's constant and Coulomb's constant only.

Recently, Faizal [19] and Pramanik et al. [20] used the Planck length to investigate the deformed Heisenberg algebra. Based on this algebra, Faizal investigated the deformation of the Wheeler-DeWitt Equation and thus showed that the big bang singularity gets obsolete. Additionally, Faizal et al. [21] adopted the idea of a minimum length scale or equivalently of a minimum time scale and showed that this leads to corrections to all quantum mechanical systems by the deformed Heisenberg algebra and thus to a discrete spectrum for time.

One of the attempts to unify general relativity with the quantum description of the microscopic cosmos has led to string theory [22] [23] [24] [25]. One of its main features is the existence of 10 or 26 space dimensions which is larger than the commonly experienced three space dimensions [26]. These extra dimensions are assumed to be compactified and are consequently too small to be observed.

On the contrary, there have not been many approaches to a second or more time dimensions. Tegmark [27] summarized that a universe with a large second time dimension cannot contain observers, like us humans, because of the lack of causality. Hence, as for the additional space dimensions in string theory, extra time dimensions need to be compactified in case of their existence. 
Multitemporal spacetime dimensions have for example been discussed by Bars and Kounnas [28] [29]; they consider two time dimensions and construct actions for interacting $p$-branes within two dimensions. They show that after a phase transition, the additional time dimension becomes part of the compactified universe. Additionally, they present a new Kaluza-Klein like dimensional reduction mechanism and propose an action for a string in two time dimensions. Due to new gauge symmetries, they observe that quantum constraints are consistent only in spacetime dimensions with signature $(25,2)$ or $(26,2)$ for a bosonic string or $(9,2)$ or $(10,2)$ in the supersymmetric case.

Chen [30] interprets two extra time dimensions as quantum hidden variables and shows that non-local properties of quantum physics or that the de Broglie wave length are natural consequences of the existence of two additional time dimensions.

We here now suggest the existence of a compactified second time dimension and subsequently derive the existence of a smallest length scale, i.e. the Planck length, and explain the constancy of the speed of light in our observable universe. Our derivations also suggest that the speed of light varied in the early universe. In Section 2, we derive the Lagrangian for a five dimensional space time parametrized with two local coordinates of the surface. On the basis of this Lagrangian, we calculate the equations of motion in Section 3 and Section 5 and derive the existence of the Planck length as well as of the constancy of the speed of light in the observable universe in Section 4 and 6. We finally conclude in Section 7.

\section{The Action in Five Dimensional Space with Two Local Time Coordinates}

In order to study the effect of five dimensional spacetime parametrized by two time coordinates on the speed of light and on the Planck length, we choose a $(2,3)$ spacetime vector

$$
x^{\mu}=\left(\begin{array}{c}
c t \\
r \cdot f\left(\frac{\gamma \tau}{\Lambda}\right) \\
\boldsymbol{x}
\end{array}\right)
$$

which is the canonical $(1,3)$ spacetime vector $(c t, \boldsymbol{x})^{\mathrm{T}}$ with

$\boldsymbol{x}=\left(x_{1}, x_{2}, x_{3}\right)^{\mathrm{T}} \in \mathbb{R}^{3}$ extended by an additional timelike coordinate

$r \cdot f(\gamma \tau / \Lambda) . \tau$ is the second time parameter, $r \in \mathbf{R}$ describes the size of the second time dimension and $\gamma$ is the characteristic velocity, thus the equivalent of $c$. $f$ describes the shape of the second time dimension and $\Lambda \in \mathbf{R}$ is a normalization parameter such that $\frac{\gamma \tau}{\Lambda}$ is dimensionless. As stated in [30], the additional time dimension has to be small compared to the first time dimension constraining $r$.

Similarly to four spacetime dimensions, the metric is given through 


$$
g^{\mu \nu}=\left(\begin{array}{rrrrr}
1 & 0 & 0 & 0 & 0 \\
0 & 1 & 0 & 0 & 0 \\
0 & 0 & -1 & 0 & 0 \\
0 & 0 & 0 & -1 & 0 \\
0 & 0 & 0 & 0 & -1
\end{array}\right)
$$

with signature $(+,+,-,-,-)$.

If we define

$$
\begin{gathered}
x_{t}^{\mu}:=\left(\begin{array}{c}
c t \\
0 \\
\eta \boldsymbol{x}
\end{array}\right), \\
x_{\tau}^{\mu}:=\left(\begin{array}{c}
0 \\
r \cdot f\left(\frac{\gamma \tau}{\Lambda}\right) \\
(1-\eta) \boldsymbol{x}
\end{array}\right)
\end{gathered}
$$

with $\eta \in(0,1)$, we can decompose $x^{\mu}$ into $x^{\mu}=x_{t}^{\mu}+x_{\tau}^{\mu}$.

Inspired by the Nambu Goto action [31] [32] which is a two dimensional integral over time and the surface of a string, we define the action as

$$
S=\int L \mathrm{~d} t \mathrm{~d} \tau
$$

As for standard electrodynamics, we make the ansatz

$$
S=\int \mathrm{d} s_{t} \mathrm{~d} s_{\tau}
$$

for $S$ where $\mathrm{ds}_{t}$ and $\mathrm{d} s_{\tau}$ describe the infinitesimal line elements along $t$ and $\tau$. It is (applying Einstein's sum convention)

$$
\begin{gathered}
\mathrm{ds}_{t}^{2}=\mathrm{d} x_{t}^{v} g_{\mu v} \mathrm{~d} x_{t}^{\mu}=[\mathrm{d}(c t)]^{2}-\eta^{2} \mathrm{~d} x^{2} \\
=[\mathrm{d} c \cdot t+c \cdot \mathrm{d} t]^{2}-\eta^{2} \mathrm{~d} x^{2} \\
=\left[\dot{c}^{2} t^{2}+c^{2}-\eta^{2} \dot{\boldsymbol{x}}^{2}+2 \dot{c} c t\right] \mathrm{d} t^{2} \\
\Rightarrow \mathrm{d} s_{t}=\sqrt{\dot{c}^{2} t^{2}+c^{2}-\eta^{2} \dot{\boldsymbol{x}}^{2}+2 \dot{\boldsymbol{c}} c t} \mathrm{~d} t
\end{gathered}
$$

where we use $\dot{c}=\mathrm{d} c / \mathrm{d} t$ and $\dot{\boldsymbol{x}}=\mathrm{d} \boldsymbol{x} / \mathrm{d} t$. Note that in the limit $c=$ const . and $\eta \rightarrow 1$, (10) becomes $\mathrm{d} s_{t}=\sqrt{c^{2}-\dot{\boldsymbol{x}}^{2}} \mathrm{~d} t$ which is the line element of a free particle in one time dimension with a constant speed of light.

Similarly we obtain:

$$
\begin{gathered}
\mathrm{d} s_{\tau}^{2}=\left[\mathrm{d}\left(r \cdot f\left(\frac{\gamma \tau}{\Lambda}\right)\right)\right]^{2}-(1-\eta)^{2} \mathrm{~d} \boldsymbol{x}^{2} \\
=r^{2}\left[\left.\frac{1}{\Lambda}(\mathrm{d} \gamma \cdot \tau+\gamma \mathrm{d} \tau) \frac{\mathrm{d} f}{\mathrm{~d} z}(z)\right|_{\left.z=\frac{\gamma t}{\Lambda}\right]^{2}-(1-\eta)^{2} \mathrm{~d} \boldsymbol{x}^{2}}\right. \\
=\frac{r^{2}}{\Lambda^{2}}\left[\left(\gamma^{\prime 2} \tau^{2}+\gamma^{2}+2 \gamma \gamma^{\prime} \tau\right) f_{z}^{2}-(1-\eta)^{2} \boldsymbol{x}^{\prime 2}\right] \mathrm{d} \tau^{2}
\end{gathered}
$$




$$
\Rightarrow \mathrm{d} s_{\tau}=\frac{r}{\Lambda} \sqrt{\left(\gamma^{\prime 2} \tau^{2}+\gamma^{2}+2 \gamma \gamma^{\prime} \tau\right) f_{z}^{2}\left(\frac{\gamma \tau}{\Lambda}\right)-(1-\eta)^{2} \boldsymbol{x}^{\prime 2}} \mathrm{~d} \tau
$$

where we use the chain rule $\mathrm{d} f\left(\frac{\gamma \tau}{\Lambda}\right)=\left.\frac{1}{\Lambda}(\mathrm{d} \gamma \cdot \tau+\gamma \mathrm{d} \tau) \frac{\mathrm{d} f}{\mathrm{~d} z}(z)\right|_{z=\frac{\gamma t}{\Lambda}}$ and define $f_{z}:=\left.\frac{\mathrm{d} f}{\mathrm{~d} z}(z)\right|_{z=\frac{\gamma t}{\Lambda}}$. The prime denotes the time derivative after $\tau: \gamma^{\prime}:=\mathrm{d} \gamma / \mathrm{d} \tau$ and $x^{\prime}:=\mathrm{d} x / \mathrm{d} \tau$.

Inserting (10) and (14) into (6) leads to

$$
S=\int_{t_{a} \tau_{a}}^{t_{e} \tau_{e}} \mathrm{~d} t \mathrm{~d} \tau \frac{r}{\Lambda} \sqrt{\dot{c}^{2} t^{2}+c^{2}-\eta^{2} \dot{\boldsymbol{x}}^{2}+2 \dot{c} c t} \times \sqrt{\left(\gamma^{\prime 2} \tau^{2}+\gamma^{2}+2 \gamma \gamma^{\prime} \tau\right) f_{z}^{2}\left(\frac{\gamma \tau}{\Lambda}\right)-(1-\eta)^{2} \mathbf{x}^{\prime 2}}
$$

By comparing (15) with (5), we identify the Lagrangian

$$
L=\frac{r}{\Lambda} \sqrt{\dot{c}^{2} t^{2}+c^{2}-\eta^{2} \dot{\boldsymbol{x}}^{2}+2 \dot{c} c t} \sqrt{\left(\gamma^{\prime 2} \tau^{2}+\gamma^{2}+2 \gamma \gamma^{\prime} \tau\right) f_{z}^{2}-(1-\eta)^{2} \boldsymbol{x}^{\prime 2}}
$$

As stated in [30], the equation of motion for a Langrangian in two time dimensions is similar to the equation of motion of a string [33], hence

$$
\frac{\mathrm{d}}{\mathrm{d} t} \frac{\partial L}{\partial \dot{x}_{i}}+\frac{\mathrm{d}}{\mathrm{d} \tau} \frac{\partial L}{\partial x_{i}^{\prime}}-\frac{\partial L}{\partial x_{i}}=0
$$

where $i$ indexes the $i$-th space dimension. In the following, we insert (16) into (17) and derive the equations of motion. Note that both $c$ and $\gamma$ also depend on $t$ or $\tau$ as well as on $x$ since they are not assumed to be constant. Subsequently, $\dot{c}$ depends on $x, \dot{x}$ and $t$ and $\gamma$ depends on $x, x^{\prime}$ and $\tau$. Hence, we here also consider the partial derivatives of $c$ and $\dot{c}$ with respect to $x$ and $\dot{x}$, similarly for $\gamma$ and $\gamma^{\prime}$. For each spatial dimension $i$, it is

$$
\begin{aligned}
0= & \frac{\mathrm{d}}{\mathrm{d} t}\left(\sqrt{\left.\frac{\left(\gamma^{\prime 2} \tau^{2}+\gamma^{2}+2 \gamma \gamma^{\prime} \tau\right) f_{z}^{2}-(1-\eta)^{2} \boldsymbol{x}^{\prime 2}}{\dot{c}^{2} t^{2}+c^{2}-\eta^{2} \dot{\boldsymbol{x}}^{2}+2 \dot{c} c t}\left(\dot{c} \frac{\partial \dot{c}}{\partial \dot{x}_{i}} t^{2}+c \frac{\partial \dot{c}}{\partial \dot{x}_{i}} t-\eta^{2} \dot{x}_{i}\right)\right)}\right. \\
& +\frac{\mathrm{d}}{\mathrm{d} \tau}\left(\sqrt{\frac{\dot{c}^{2} t^{2}+c^{2}-\eta^{2} \dot{\boldsymbol{x}}^{2}+2 \dot{c} c t}{\left(\gamma^{\prime 2} \tau^{2}+\gamma^{2}+2 \gamma \gamma^{\prime} \tau\right) f_{z}^{2}-(1-\eta)^{2} \boldsymbol{x}^{\prime 2}}}\right. \\
& \left.\times\left(\left(\gamma^{\prime} \frac{\partial \gamma^{\prime}}{\partial x_{i}^{\prime}} \tau^{2}+\gamma \frac{\partial \gamma^{\prime}}{\partial x_{i}^{\prime}} \tau\right) f_{z}^{2}-(1-\eta)^{2} x_{i}^{\prime}\right)\right) \\
& -\frac{\partial}{\partial x_{i}}\left(\sqrt{\dot{c}^{2} t^{2}+c^{2}-\eta^{2} \dot{\boldsymbol{x}}^{2}+2 \dot{c} c t}\right. \\
& \left.\times \sqrt{\left(\gamma^{\prime 2} \tau^{2}+\gamma^{2}+2 \gamma \gamma^{\prime} \tau\right) f_{z}^{2}-(1-\eta)^{2} \boldsymbol{x}^{\prime 2}}\right)
\end{aligned}
$$

For simplicity we assume that $x^{\prime}$ is independent of $t$ and $\dot{x}$ is independent of $\tau$. We define

$$
\begin{gathered}
\mathcal{F}_{1}(t):=(\dot{c} t+c)^{2}-\eta^{2} \dot{\boldsymbol{x}}^{2} \\
\mathcal{F}_{2}(\tau):=\left(\gamma^{\prime} \tau+\gamma\right)^{2} f_{z}^{2}\left(\frac{\gamma \tau}{\Lambda}\right)-(1-\eta)^{2} \boldsymbol{x}^{\prime 2}
\end{gathered}
$$


and rewrite Equation (18) as

$$
\begin{aligned}
0= & \frac{\mathrm{d}}{\mathrm{d} t}\left(\sqrt{\frac{\mathcal{F}_{2}(\tau)}{\mathcal{F}_{1}(t)}}\left(\dot{c} \frac{\partial \dot{c}}{\partial \dot{x}_{i}} t^{2}+c \frac{\partial \dot{c}}{\partial \dot{x}_{i}} t-\eta^{2} \dot{x}_{i}\right)\right) \\
& +\frac{\mathrm{d}}{\mathrm{d} \tau}\left(\sqrt{\frac{\mathcal{F}_{1}(t)}{\mathcal{F}_{2}(\tau)}}\left(\left(\gamma^{\prime} \frac{\partial \gamma^{\prime}}{\partial x_{i}^{\prime}} \tau^{2}+\gamma \frac{\partial \gamma^{\prime}}{\partial x_{i}^{\prime}} \tau\right) f_{z}^{2}-(1-\eta)^{2} x_{i}^{\prime}\right)\right) \\
& -\frac{1}{2} \sqrt{\frac{\mathcal{F}_{2}(\tau)}{\mathcal{F}_{1}(t)}} \frac{\partial \mathcal{F}_{1}(t)}{\partial x_{i}}-\frac{1}{2} \sqrt{\frac{\mathcal{F}_{1}(t)}{\mathcal{F}_{2}(\tau)}} \frac{\partial \mathcal{F}_{2}(\tau)}{\partial x_{i}}
\end{aligned}
$$

Dividing (21) by $\sqrt{\mathcal{F}_{1}(t) \mathcal{F}_{2}(\tau)}$ yields

$$
\begin{aligned}
0= & \frac{1}{\sqrt{\mathcal{F}_{1}(t)}} \frac{\mathrm{d}}{\mathrm{d} t}\left(\frac{1}{\sqrt{\mathcal{F}_{1}(t)}}\left(\dot{c} \frac{\partial \dot{c}}{\partial \dot{x}_{i}} t^{2}+c \frac{\partial \dot{c}}{\partial \dot{x}_{i}} t-\eta^{2} \dot{x}_{i}\right)\right) \\
& +\frac{1}{\sqrt{\mathcal{F}_{2}(\tau)}} \frac{\mathrm{d}}{\mathrm{d} \tau}\left(\frac{1}{\sqrt{\mathcal{F}_{2}(\tau)}}\left(\left(\gamma^{\prime} \frac{\partial \gamma^{\prime}}{\partial x_{i}^{\prime}} \tau^{2}+\gamma \frac{\partial \gamma^{\prime}}{\partial x_{i}^{\prime}} \tau\right) f_{z}^{2}-(1-\eta)^{2} x_{i}^{\prime}\right)\right) \\
& -\frac{1}{2} \frac{1}{\mathcal{F}_{1}(t)} \frac{\partial \mathcal{F}_{1}(t)}{\partial x_{i}}-\frac{1}{2} \frac{1}{\mathcal{F}_{2}(\tau)} \frac{\partial \mathcal{F}_{2}(\tau)}{\partial x_{i}} \\
\Leftrightarrow & \frac{1}{\sqrt{\mathcal{F}_{1}(t)}} \frac{\mathrm{d}}{\mathrm{d} t}\left(\frac{1}{\sqrt{\mathcal{F}_{1}(t)}}\left(\dot{c} \frac{\partial \dot{c}}{\partial \dot{x}_{i}} t^{2}+c \frac{\partial \dot{c}}{\partial \dot{x}_{i}} t-\eta^{2} \dot{x}_{i}\right)\right)-\frac{1}{2} \frac{1}{\mathcal{F}_{1}(t)} \frac{\partial \mathcal{F}_{1}(t)}{\partial x_{i}} \\
= & -\frac{1}{\sqrt{\mathcal{F}_{2}(\tau)}} \frac{\mathrm{d}}{\mathrm{d} \tau}\left(\frac{1}{\sqrt{\mathcal{F}_{2}(\tau)}}\left(\left(\gamma^{\prime} \frac{\partial \gamma^{\prime}}{\partial x_{i}^{\prime}} \tau^{2}+\gamma \frac{\partial \gamma^{\prime}}{\partial x_{i}^{\prime}} \tau\right) f_{z}^{2}-(1-\eta)^{2} x_{i}^{\prime}\right)\right) \\
& +\frac{1}{2} \frac{1}{\mathcal{F}_{2}(\tau)} \frac{\partial \mathcal{F}_{2}(\tau)}{\partial x_{i}}
\end{aligned}
$$

\section{The Equation of Motion for the Second Time Dimension}

Since (23) holds for all $t$ on the left-hand-side and for all $\tau$ on the right-hand-side, both sides have to be equal to a constant $-\Omega_{i}$ :

$$
\begin{aligned}
& \frac{1}{\sqrt{\mathcal{F}_{1}(t)}} \frac{\mathrm{d}}{\mathrm{d} t}\left(\frac{1}{\sqrt{\mathcal{F}_{1}(t)}}\left(\dot{c} \frac{\partial \dot{c}}{\partial \dot{x}_{i}} t^{2}+c \frac{\partial \dot{c}}{\partial \dot{x}_{i}} t-\eta^{2} \dot{x}_{i}\right)\right)-\frac{1}{2} \frac{1}{\mathcal{F}_{1}(t)} \frac{\partial \mathcal{F}_{1}(t)}{\partial x_{i}} \\
& =-\frac{1}{\sqrt{\mathcal{F}_{2}(\tau)}} \frac{\mathrm{d}}{\mathrm{d} \tau}\left(\frac{1}{\sqrt{\mathcal{F}_{2}(\tau)}}\left(\left(\gamma^{\prime} \frac{\partial \gamma^{\prime}}{\partial x_{i}^{\prime}} \tau^{2}+\gamma \frac{\partial \gamma^{\prime}}{\partial x_{i}^{\prime}} \tau\right) f_{z}^{2}-(1-\eta)^{2} x_{i}^{\prime}\right)\right) \\
& +\frac{1}{2} \frac{1}{\mathcal{F}_{2}(\tau)} \frac{\partial \mathcal{F}_{2}(\tau)}{\partial x_{i}}=-\Omega_{i}
\end{aligned}
$$

We now investigate both sides separately. For the time dimension $\tau$ it is thus

$$
\begin{aligned}
& -\frac{1}{\sqrt{\mathcal{F}_{2}(\tau)}} \frac{\mathrm{d}}{\mathrm{d} \tau}\left(\frac{1}{\sqrt{\mathcal{F}_{2}(\tau)}}\left(\left(\gamma^{\prime} \frac{\partial \gamma^{\prime}}{\partial x_{i}^{\prime}} \tau^{2}+\gamma \frac{\partial \gamma^{\prime}}{\partial x_{i}^{\prime}} \tau\right) f_{z}^{2}-(1-\eta)^{2} x_{i}^{\prime}\right)\right) \\
& =-\Omega_{i}-\frac{1}{2} \frac{1}{\mathcal{F}_{2}(\tau)} \frac{\partial \mathcal{F}_{2}(\tau)}{\partial x_{i}}
\end{aligned}
$$


Multiplying both sides with $\left(\gamma^{\prime} \frac{\partial \gamma^{\prime}}{\partial x_{i}^{\prime}} \tau^{2}+\gamma \frac{\partial \gamma^{\prime}}{\partial x_{i}^{\prime}} \tau\right) f_{z}^{2}-(1-\eta)^{2} x_{i}^{\prime}$ yields

$$
\begin{aligned}
- & \frac{\left(\gamma^{\prime} \frac{\partial \gamma^{\prime}}{\partial x_{i}^{\prime}} \tau^{2}+\gamma \frac{\partial \gamma^{\prime}}{\partial x_{i}^{\prime}} \tau\right) f_{z}^{2}-(1-\eta)^{2} x_{i}^{\prime}}{\sqrt{\mathcal{F}_{2}(\tau)}} \\
\times & \frac{\mathrm{d}}{\mathrm{d} \tau}\left(\frac{1}{\sqrt{\mathcal{F}_{2}(\tau)}}\left(\left(\gamma^{\prime} \frac{\partial \gamma^{\prime}}{\partial x_{i}^{\prime}} \tau^{2}+\gamma \frac{\partial \gamma^{\prime}}{\partial x_{i}^{\prime}} \tau\right) f_{z}^{2}-(1-\eta)^{2} x_{i}^{\prime}\right)\right) \\
= & \Omega_{i}(1-\eta)^{2} x_{i}^{\prime}-\Omega_{i}\left(\gamma^{\prime} \frac{\partial \gamma^{\prime}}{\partial x_{i}^{\prime}} \tau^{2}+\gamma \frac{\partial \gamma^{\prime}}{\partial x_{i}^{\prime}} \tau\right) f_{z}^{2} \\
& -\frac{1}{2} \frac{1}{\mathcal{F}_{2}(\tau)} \frac{\partial \mathcal{F}_{2}(\tau)}{\partial x_{i}}\left(\left(\gamma^{\prime} \frac{\partial \gamma^{\prime}}{\partial x_{i}^{\prime}} \tau^{2}+\gamma \frac{\partial \gamma^{\prime}}{\partial x_{i}^{\prime}} \tau\right) f_{z}^{2}-(1-\eta)^{2} x_{i}^{\prime}\right) \\
\Leftrightarrow & -\frac{1}{2} \frac{\mathrm{d}}{\mathrm{d} \tau}\left(\frac{1}{\mathcal{F}_{2}(\tau)}\left(\left(\gamma^{\prime} \frac{\partial \gamma^{\prime}}{\partial x_{i}^{\prime}} \tau^{2}+\gamma \frac{\partial \gamma^{\prime}}{\partial x_{i}^{\prime}} \tau\right) f_{z}^{2}-(1-\eta)^{2} x_{i}^{\prime}\right)^{2}\right) \\
= & \Omega_{i}(1-\eta)^{2} x_{i}^{\prime}-\Omega_{i}\left(\gamma^{\prime} \frac{\partial \gamma^{\prime}}{\partial x_{i}^{\prime}} \tau^{2}+\gamma \frac{\partial \gamma^{\prime}}{\partial x_{i}^{\prime}} \tau\right) f_{z}^{2} \\
& -\frac{1}{2} \frac{1}{\mathcal{F}_{2}(\tau)} \frac{\partial \mathcal{F}_{2}(\tau)}{\partial x_{i}}\left(\left(\gamma^{\prime} \frac{\partial \gamma^{\prime}}{\partial x_{i}^{\prime}} \tau^{2}+\gamma \frac{\partial \gamma^{\prime}}{\partial x_{i}^{\prime}} \tau\right) f_{z}^{2}-(1-\eta)^{2} x_{i}^{\prime}\right)
\end{aligned}
$$

Integrating both sides then gives

$$
\begin{aligned}
& -\frac{1}{2} \frac{1}{\mathcal{F}_{2}(\tau)}\left(\left(\gamma^{\prime} \frac{\partial \gamma^{\prime}}{\partial x_{i}^{\prime}} \tau^{2}+\gamma \frac{\partial \gamma^{\prime}}{\partial x_{i}^{\prime}} \tau\right) f_{z}^{2}-(1-\eta)^{2} x_{i}^{\prime}\right)^{2} \\
& =\Omega_{i}(1-\eta)^{2} x_{i}+I_{i, 3}+S_{12}\left(x_{i}, x_{i}^{\prime}, \tau\right)
\end{aligned}
$$

where $I_{i, 3}$ is an integration constant and where we have defined

$$
S_{12}\left(x_{i}, x_{i}^{\prime}, \tau\right):=\int \mathrm{d} \tau\left(S_{1}+S_{2}\right)
$$

with

$$
\begin{gathered}
S_{1}\left(x_{i}, x_{i}^{\prime}, \tau\right):=-\Omega_{i}\left(\gamma^{\prime} \frac{\partial \gamma^{\prime}}{\partial x_{i}^{\prime}} \tau^{2}+\gamma \frac{\partial \gamma^{\prime}}{\partial x_{i}^{\prime}} \tau\right) f_{z}^{2} \\
S_{2}\left(x_{i}, x_{i}^{\prime}, \tau\right):=\frac{1}{2} \frac{1}{\mathcal{F}_{2}(\tau)} \frac{\partial \mathcal{F}_{2}(\tau)}{\partial x_{i}}\left(\left(\gamma^{\prime} \frac{\partial \gamma^{\prime}}{\partial x_{i}^{\prime}} \tau^{2}+\gamma \frac{\partial \gamma^{\prime}}{\partial x_{i}^{\prime}} \tau\right) f_{z}^{2}-(1-\eta)^{2} x_{i}^{\prime}\right)
\end{gathered}
$$

Multiplying (28) with $-2 \mathcal{F}_{2}(\tau)$ then gives

$$
\begin{aligned}
& \left(\left(\gamma^{\prime} \frac{\partial \gamma^{\prime}}{\partial x_{i}^{\prime}} \tau^{2}+\gamma \frac{\partial \gamma^{\prime}}{\partial x_{i}^{\prime}} \tau\right) f_{z}^{2}-(1-\eta)^{2} x_{i}^{\prime}\right)^{2} \\
& =2\left(-\Omega_{i}(1-\eta)^{2} x_{i}-I_{i, 3}\right)\left(\gamma^{\prime} \tau+\gamma\right)^{2} f_{z}^{2}-2\left(-\Omega_{i}(1-\eta)^{2} x_{i}-I_{i, 3}\right)(1-\eta)^{2} x_{i}^{\prime 2} \\
& -2 \mathcal{F}_{2}(\tau) S_{12}\left(x_{i}, x_{i}^{\prime}, \tau\right)
\end{aligned}
$$

where we assume for simplicity, but without loss of generality, that a test particle moves in one of the directions $i$ only. 
Defining further

$$
\begin{aligned}
S\left(x_{i}, x_{i}^{\prime}, \tau\right):= & -2 \mathcal{F}_{2} S_{12}-\left(\gamma^{\prime} \frac{\partial \gamma^{\prime}}{\partial x_{i}^{\prime}} \tau^{2}+\gamma \frac{\partial \gamma^{\prime}}{\partial x_{i}^{\prime}} \tau\right)^{2} f_{z}^{4} \\
& +2\left(\gamma^{\prime} \frac{\partial \gamma^{\prime}}{\partial x_{i}^{\prime}} \tau^{2}+\gamma \frac{\partial \gamma^{\prime}}{\partial x_{i}^{\prime}} \tau\right) f_{z}^{2}(1-\eta)^{2} x_{i}^{\prime}
\end{aligned}
$$

we can further simplify (32) to

$$
\begin{aligned}
(1-\eta)^{4} x_{i}^{\prime 2}= & 2\left(-\Omega_{i}(1-\eta)^{2} x_{i}-I_{i, 3}\right)\left(\gamma^{\prime} \tau+\gamma\right)^{2} f_{z}^{2} \\
& -2\left(-\Omega_{i}(1-\eta)^{2} x_{i}-I_{i, 3}\right)(1-\eta)^{2} x_{i}^{\prime 2}+S
\end{aligned}
$$

which is equivalent to

$$
\begin{aligned}
& \mathrm{d} x_{i}(1-\eta) \sqrt{1+\frac{1}{-2\left(\Omega_{i} x_{i}+\frac{I_{i, 3}}{(1-\eta)^{2}}\right)}} \\
& =\mathrm{d} \tau \sqrt{\left(\gamma^{\prime} \tau+\gamma\right)^{2} f_{z}^{2}+\frac{S}{-2\left(\Omega_{i}(1-\eta)^{2} x_{i}+I_{i, 3}\right)}} \\
& =\mathrm{d} \tau\left(\gamma^{\prime} \tau+\gamma\right) f_{z}+\mathrm{d} \tau\left[\frac{S}{-4\left(\Omega_{i}(1-\eta)^{2} x_{i}+I_{i, 3}\right)\left(\gamma^{\prime} \tau+\gamma\right) f_{z}} \mp \cdots\right]
\end{aligned}
$$

where we used the series expansion $\sqrt{x+a}=\sqrt{a}+x /(2 \sqrt{a}) \mp \cdots$ for the last equality.

Finally integrating both sides of (35) gives an implicit solution for $x_{i}$ :

$$
\left.\begin{array}{rl}
\frac{1-\eta}{\sqrt{2 \Omega_{i}}}\left[\sqrt{2 \Omega_{i}\left(\frac{\left.\Omega_{i} x_{i}+\frac{I_{i, 3}}{(1-\eta)^{2}}\right)^{2}}{\Omega_{i}}-\frac{\Omega_{i} x_{i}+\frac{I_{i, 3}}{(1-\eta)^{2}}}{\Omega_{i}}\right.}\right. \\
\left.-\frac{1}{\sqrt{8 \Omega}} \ln \left(\sqrt{\left.4 \Omega_{i}^{2}\left(4 \Omega_{i} x_{i}+\frac{4 I_{i, 3}}{(1-\eta)^{2}}-1\right)^{2}-1+8 \Omega_{i}^{2} x_{i}+8 \Omega_{i} \frac{I_{i, 3}}{(1-\eta)^{2}}-2 \Omega_{i}\right]}\right)\right] \\
=\frac{1}{\Lambda} f\left(\frac{\gamma \tau}{\Lambda}\right)+\int \mathrm{d} \tau\left[\frac{S}{-4\left(\Omega_{i}(1-\eta)^{2} x_{i}+I_{i, 3}\right)\left(\gamma^{\prime} \tau+\gamma\right) f_{z}}\right]+I_{i, 4}
\end{array}\right]
$$

where $I_{i, 4}$ is another integration constant.

\section{The Existence of the Planck Length}

Since the solution (36) has to be physical, the argument of the logarithm must be positive:

$$
\sqrt{4 \Omega_{i}^{2}\left(4 \Omega_{i} x_{i}+\frac{4 I_{i, 3}}{(1-\eta)^{2}}-1\right)^{2}}-1+8 \Omega_{i}^{2} x_{i}+8 \Omega_{i} \frac{I_{i, 3}}{(1-\eta)^{2}}-2 \Omega_{i}>0
$$




$$
\begin{gathered}
\sqrt{4 \Omega_{i}^{2}\left(4 \Omega_{i} z-1\right)^{2}-1}+8 \Omega_{i}^{2} z-2 \Omega_{i}>0 \\
\sqrt{4 \Omega_{i}^{2}\left(4 \Omega_{i} z-1\right)^{2}-1}>-8 \Omega_{i}^{2} z+2 \Omega_{i}
\end{gathered}
$$

with the definition

$$
\mathrm{z}:=\frac{\Omega_{i} x_{i}+\frac{I_{i, 3}}{(1-\eta)^{2}}}{\Omega_{i}} .
$$

We will now prove by contradiction that for the second time dimension it is

$$
-8 \Omega_{i}^{2} z+2 \Omega_{i}<0 .
$$

Let us therefore assume that $-8 \Omega_{i}^{2} z+2 \Omega_{i} \geq 0$. Squaring

$$
\sqrt{4 \Omega_{i}^{2}\left(4 \Omega_{i} z-1\right)^{2}-1}>-8 \Omega_{i}^{2} z+2 \Omega_{i} .
$$

yields

$$
4 \Omega_{i}^{2}\left(4 \Omega_{i} z-1\right)^{2}-1>\left(-8 \Omega_{i}^{2} z+2 \Omega_{i}\right)^{2}=4 \Omega_{i}^{2}\left(4 \Omega_{i} z-1\right)^{2}
$$

Subtracting $4 \Omega_{i}^{2}\left(4 \Omega_{i} z-1\right)^{2}$ gives

$$
-1>0
$$

which is obviously wrong. Thus $-8 \Omega_{i}^{2} z+2 \Omega_{i}$ must be negative; this leads to

$$
\begin{gathered}
-8 \Omega_{i}^{2} z+2 \Omega_{i}<0 \\
\Leftrightarrow 2 \Omega_{i}\left(-4 \Omega_{i} z+1\right)<0 \\
\Leftrightarrow\left(2 \Omega_{i}<0 \wedge\left(-4 \Omega_{i} z+1\right)>0\right) \vee\left(2 \Omega_{i}>0 \wedge\left(-4 \Omega_{i} z+1\right)<0\right) \\
\Leftrightarrow\left(\Omega_{i}<0 \wedge 4 \Omega_{i} z<1\right) \vee\left(\Omega_{i}>0 \wedge 4 \Omega_{i} z>1\right) \\
\Leftrightarrow\left(z>\frac{1}{4 \Omega_{i}} \text { for } \Omega_{i}<0\right) \vee\left(z>\frac{1}{4 \Omega_{i}} \text { for } \Omega_{i}>0\right) \\
\Leftrightarrow z>\frac{1}{4 \Omega_{i}} \\
\Leftrightarrow x_{i}>\frac{1}{4 \Omega_{i}}\left(1-\frac{I_{i, 3}}{(1-\eta)^{2}}\right) \forall \Omega_{i} \neq 0
\end{gathered}
$$

where we used that a product of two factors is smaller than 0 iff one of the factors is larger than 0 and the other one is smaller than $0 . I_{i, 3}$ is determined by (28)

$$
\begin{aligned}
& -\frac{1}{2} \frac{1}{\mathcal{F}_{2}(\tau)}\left(\left(\gamma^{\prime} \frac{\partial \gamma^{\prime}}{\partial x_{i}^{\prime}} \tau^{2}+\gamma \frac{\partial \gamma^{\prime}}{\partial x_{i}^{\prime}} \tau\right) f_{z}^{2}-(1-\eta)^{2} x_{i}^{\prime}\right)^{2} \\
& =\Omega_{i}(1-\eta)^{2} x_{i}+I_{i, 2}+S_{12}\left(x_{i}, x_{i}^{\prime}, \tau\right)
\end{aligned}
$$


Given the initial conditions $x_{i}^{\prime}\left(\tau_{0}\right)=: x_{k, 0}^{\prime}$ and $x_{k}\left(t_{0}, \tau_{0}\right)=: x_{k, 0}$, it is

$$
\begin{gathered}
-\frac{I_{i, 3}}{(1-\eta)^{2}}=\Omega_{i} x_{k, 0}+\frac{S_{12}\left(x_{k, 0}, x_{k, 0}^{\prime}, \tau_{0}\right)}{(1-\eta)^{2}} \\
+\frac{1}{2(1-\eta)^{2}} \frac{1}{\mathcal{F}_{2}\left(\tau_{0}\right)}\left(\left.\left(\gamma^{\prime}\left(\tau_{0}\right) \frac{\partial \gamma^{\prime}}{\partial x_{i}^{\prime}}\left(\tau_{0}\right) \tau_{0}^{2}+\gamma\left(\tau_{0}\right) \frac{\partial \gamma^{\prime}}{\partial x_{i}^{\prime}}\left(\tau_{0}\right) \tau_{0}\right) f_{z}^{2}\right|_{z=\frac{\gamma\left(\tau_{0}\right) \tau_{0}}{\Lambda}}\right. \\
\left.-(1-\eta)^{2} x_{k, 0}^{\prime}\right)^{2} \\
-\frac{I_{i, 3}}{(1-\eta)^{2}}=: \Omega_{i} x_{k, 0}+\mathcal{R}
\end{gathered}
$$

For some functions $f$ and for some parameters $\{\gamma, \Lambda\}$, i.e. for some particular shapes of the second time dimension, we can assume $\mathcal{R} / \Omega_{i}>0$ and hence, inserting (81) into (78),

$$
x_{i}>\frac{1}{4 \Omega_{i}}\left(1+\Omega_{i} x_{k, 0}+\mathcal{R}\right)=\frac{1}{4 \Omega_{i}}+\frac{x_{k, 0}}{4}+\frac{\mathcal{R}}{4 \Omega_{i}}>\frac{1}{4 \Omega_{i}}
$$

which implies that

$$
\left|x_{i}\right|>\frac{1}{4\left|\Omega_{i}\right|}
$$

for $\Omega_{i}>0$.

Equation (56) states that there is always a lower threshold for any coordinate; thus, the existence of a second time dimension naturally explains the existence of the so-called Planck-length, i.e. a smallest length scale. Hence we identify $1 /\left(4 \Omega_{i}\right)$ being in the order of the Planck scale $\ell_{P} \approx 10^{-35} \mathrm{~m}$, and thus, for the observable universe it is $1 /\left(4 \Omega_{i}\right) \rightarrow 0$, or equivalently $\Omega_{i} \rightarrow \infty$.

\section{The Equation of Motion for the First Time Dimension}

We now come back to analyse the equation of motion for the first time dimension. The first part of (24) leads to the equation

$$
\begin{aligned}
& \frac{1}{\sqrt{\mathcal{F}_{1}(t)}} \frac{\mathrm{d}}{\mathrm{d} t}\left(\frac{1}{\sqrt{\mathcal{F}_{1}(t)}}\left(\dot{c} \frac{\partial \dot{c}}{\partial \dot{x}_{i}} t^{2}+c \frac{\partial \dot{c}}{\partial \dot{x}_{i}} t-\eta^{2} \dot{x}_{i}\right)\right) \\
& =-\Omega_{i}+\frac{1}{2} \frac{1}{\mathcal{F}_{1}(t)} \frac{\partial \mathcal{F}_{1}(t)}{\partial x_{i}}
\end{aligned}
$$

For the observable universe, i.e. $\Omega_{i} \rightarrow \infty$ the right hand side of (57) is governed by large $\Omega_{i}$, thus ${ }^{1}$

$$
\frac{1}{\sqrt{\mathcal{F}_{1}(t)}} \frac{\mathrm{d}}{\mathrm{d} t}\left(\frac{1}{\sqrt{\mathcal{F}_{1}(t)}}\left(\dot{c} \frac{\partial \dot{c}}{\partial \dot{x}_{i}} t^{2}+c \frac{\partial \dot{c}}{\partial \dot{x}_{i}} t-\eta^{2} \dot{x}_{i}\right)\right) \simeq-\Omega_{i}
$$

${ }^{1}$ The dominance of $\Omega_{i}$ can easily be seen by multiplying (57) with $\sqrt{\mathcal{F}_{1}}$ and by inserting the ansatz $\sqrt{\mathcal{F}_{1}} \equiv u\left(x_{i}, \dot{x}_{i}, t\right):=\mathrm{e}^{k k_{i}+a t+v \dot{x}}$ for arbitrary $k$. 
Multiplying both sides with $\dot{c} \frac{\partial \dot{c}}{\partial \dot{x}_{i}} t^{2}+c \frac{\partial \dot{c}}{\partial \dot{x}_{i}} t-\eta^{2} \dot{x}_{i}$ yields

$$
\frac{1}{2} \frac{\mathrm{d}}{\mathrm{d} t}\left(\frac{\left(\dot{c} \frac{\partial \dot{c}}{\partial \dot{x}_{i}} t^{2}+c \frac{\partial \dot{c}}{\partial \dot{x}_{i}} t-\eta^{2} \dot{x}_{i}\right)^{2}}{\mathcal{F}_{1}(t)}\right)=\Omega \eta^{2} \dot{x}_{i}-\Omega_{i}\left(\dot{c} \frac{\partial \dot{c}}{\partial \dot{x}_{i}} t^{2}+c \frac{\partial \dot{c}}{\partial \dot{x}_{i}} t\right)
$$

and integrating both sides over $t$ we obtain

$$
\begin{aligned}
& \frac{1}{2} \frac{\left(\dot{c} \frac{\partial \dot{c}}{\partial \dot{x}_{i}} t^{2}+c \frac{\partial \dot{c}}{\partial \dot{x}_{i}} t-\eta^{2} \dot{x}_{i}\right)^{2}}{\mathcal{F}_{1}(t)} \\
& =\Omega \eta^{2} x_{i}+I_{i, 1}-\Omega_{i} \int \mathrm{d} t\left(\dot{c} \frac{\partial \dot{c}}{\partial \dot{x}_{i}} t^{2}+c \frac{\partial \dot{c}}{\partial \dot{x}_{i}} t\right)
\end{aligned}
$$

where $I_{i, 1}$ is an integration constant. Integrating by parts and using $\int \frac{\partial \dot{c}}{\partial \dot{x}_{i}} \mathrm{~d} t=\frac{\partial}{\partial \dot{x}_{i}} \int \dot{c} \mathrm{~d} t=0$, since $c$ is independent of $\dot{x}_{i}$, makes the integral on the right-hand-side vanish, hence

$$
\frac{1}{2} \frac{\left(\dot{c} \frac{\partial \dot{c}}{\partial \dot{x}_{i}} t^{2}+c \frac{\partial \dot{c}}{\partial \dot{x}_{i}} t-\eta^{2} \dot{x}_{i}\right)^{2}}{\mathcal{F}_{1}(t)}=\Omega \eta^{2} x_{i}+I_{i, 1}
$$

For simplicity, but without loss of generality, we now assume a test particle moving in one of the three spatial directions $k$ only. Considering again integration by parts for all terms containing $\partial \dot{c} / \partial \dot{x}_{k}$ and rescaling $\Omega_{k} / \eta^{2} \rightarrow \Omega_{k}$ the solution of (61) is given by

$$
\begin{aligned}
& \frac{4}{\sqrt{8 \Omega_{k}}} \sqrt{2 \Omega_{k} x_{k}+2 I_{k, 1}+\left(\Omega_{k} x_{k}+I_{k, 1}\right)^{2}} \\
& +\frac{2}{\sqrt{2 \Omega_{k}}} \ln \left(\sqrt{4 \Omega_{k}^{2}\left(\Omega_{k} x_{k}+I_{k, 1}+1\right)^{2}-1}+2 \Omega_{k}^{2} x_{k}+2 \Omega_{k} I_{k, 1}+2 \Omega_{k}\right) \\
& =2 \sqrt{2 \Omega_{k}} c t+I_{k, 2}
\end{aligned}
$$

with another integration constant $I_{k, 2}$.

\section{The Constancy of the Speed of Light}

In this section, we show that the existence of the Planck length $\ell_{p} \sim \frac{1}{\Omega_{k}}$ and the subsequent limit $\Omega_{k} \rightarrow \infty$ implies the constancy of the speed of light for the observable universe.

Inserting $x_{k}\left(t_{0}, \tau_{0}\right)=x_{k, 0}$ into (62) gives

$$
\begin{aligned}
I_{k, 2}= & 2 \sqrt{2 \Omega_{k}} c t_{0}+\frac{4}{\sqrt{8 \Omega_{k}}} \sqrt{2 \Omega_{k} x_{k, 0}+2 I_{k, 1}+\left(\Omega_{k} x_{k, 0}+I_{k, 1}\right)^{2}} \\
& +\frac{2}{\sqrt{2 \Omega_{k}}} \ln \left(\sqrt{4 \Omega_{k}^{2}\left(\Omega_{k} x_{k, 0}+I_{k, 1}+1\right)^{2}-1}+2 \Omega_{k}^{2} x_{k, 0}+2 \Omega_{k} I_{k, 1}+2 \Omega_{k}\right)
\end{aligned}
$$


Inserting (63) further into (62) and solving for $c$ yields

$$
\begin{aligned}
c= & \frac{1}{t-t_{0}}\left[\frac { 1 } { 2 \Omega _ { k } } \left(\sqrt{2 \Omega_{k} x_{k}+2 I_{k, 1}+\left(\Omega_{k} x_{k}+I_{k, 1}\right)^{2}}\right.\right. \\
& \left.-\sqrt{2 \Omega_{k} x_{k, 0}+2 I_{k, 1}+\left(\Omega_{k} x_{k, 0}+I_{k, 1}\right)^{2}}\right) \\
& \left.+\frac{1}{2 \sqrt{2} \Omega_{k}} \ln \left(\frac{\sqrt{4 \Omega_{k}^{2}\left(\Omega_{k} x_{k}+I_{k, 1}+1\right)^{2}-1}+2 \Omega_{k}^{2} x_{k}+2 \Omega_{k} I_{k, 1}+2 \Omega_{k}}{\sqrt{4 \Omega_{k}^{2}\left(\Omega_{k} x_{k, 0}+I_{k, 1}+1\right)^{2}-1}+2 \Omega_{k}^{2} x_{k, 0}+2 \Omega_{k} I_{k, 1}+2 \Omega_{k}}\right)\right]
\end{aligned}
$$

Subsequently it is

$$
\begin{aligned}
\dot{c}= & -\frac{1}{\left(t-t_{0}\right)^{2}}\left[\frac { 1 } { 2 \Omega _ { k } } \left(\sqrt{2 \Omega_{k} x_{k}+2 I_{k, 1}+\left(\Omega_{k} x_{k}+I_{k, 1}\right)^{2}}\right.\right. \\
& \left.-\sqrt{2 \Omega_{k} x_{k, 0}+2 I_{k, 1}+\left(\Omega_{k} x_{k, 0}+I_{k, 1}\right)^{2}}\right) \\
& \left.+\frac{1}{2 \sqrt{2} \Omega_{k}} \ln \left(\frac{\sqrt{4 \Omega_{k}^{2}\left(\Omega_{k} x_{k, 0}+I_{k, 1}+1\right)^{2}-1}+2 \Omega_{k}^{2} x_{k, 0}+2 \Omega_{k} I_{k, 1}+2 \Omega_{k}}{\sqrt{4 \Omega_{k}^{2}\left(\Omega_{k} x_{k, 0}+I_{k, 1}+1\right)^{2}-1}+2 \Omega_{k}^{2} x_{k, 0}+2 \Omega_{k} I_{k, 1}+2 \Omega_{k}}\right)\right] \\
& +\frac{1}{t-t_{0}}\left[\frac{1}{2 \Omega_{k}} \frac{2 \Omega_{k} \dot{x}_{k}+2 \Omega_{k} \dot{x}_{k}\left(\Omega_{k} x_{k}+I_{k, 1}\right)}{2 \sqrt{2 \Omega_{k} x_{k}+2 I_{k, 1}+\left(\Omega_{k} x_{k}+I_{k, 1}\right)^{2}}}\right. \\
& +\frac{1}{2 \sqrt{2} \Omega_{k}} \frac{1}{\sqrt{4 \Omega_{k}^{2}\left(\Omega_{k} x_{k}+I_{k, 1}+1\right)^{2}-1}+2 \Omega_{k}^{2} x_{k}+2 \Omega_{k} I_{k, 1}+2 \Omega_{k}} \\
& \left.\times\left(\frac{4 \Omega_{k}^{3} \dot{x}_{k}\left(\Omega_{k} x_{k}+I_{k, 1}+1\right)}{\sqrt{4 \Omega_{k}^{2}\left(\Omega_{k} x_{k}+I_{k, 1}+1\right)^{2}-1}+2 \Omega_{k}^{2} \dot{x}_{k}}\right)\right]
\end{aligned}
$$

and further, taking the limit $\Omega_{k} \rightarrow \infty$ :

$$
\dot{c} \rightarrow \frac{1}{2} \frac{1}{t-t_{0}}\left(\dot{x}_{k}-\frac{x_{k}-x_{k, 0}}{t-t_{0}}\right)=: \dot{c}_{\infty}(t) .
$$

We now show by induction that for $\Omega_{k} \rightarrow \infty$ it is $c_{\infty}^{(n)}\left(t_{0}\right)=0 \forall n \geq 1$ where $c_{\infty}^{(n)}$ denotes the $n$-th derivative of $c_{\infty}$ :

1) Base case ( $n=1$ and $n=2)$ :

With the rule of L'Hôpital, it follows that

$$
\dot{c}_{\infty}\left(t_{0}\right)=\lim _{t \rightarrow t_{0}} \dot{c}_{\infty}(t)=\frac{1}{4} \ddot{X}_{k}\left(t_{0}\right) .
$$

For $\Omega_{k} \rightarrow \infty$ (61) becomes

$$
\dot{x}_{k}=2\left(\dot{c}_{\infty} t+c_{\infty}\right)
$$

with derivative

$$
\ddot{x}_{k}(t)=2 \ddot{c}_{\infty} t+4 \dot{c}_{\infty} .
$$


Inserting (67) into (69) then gives

$$
\ddot{c}_{\infty}\left(t_{0}\right)=0 .
$$

Note that the derivative of (66) leads to

$$
\ddot{c}_{\infty}(t)=\frac{1}{2} \frac{\ddot{x}(t)}{t-t_{0}}
$$

which is equivalent to $\ddot{x}(t)=2 \ddot{c}_{\infty} \cdot\left(t-t_{0}\right)$ and thus

$$
\ddot{x}\left(t_{0}\right)=0
$$

because of (70), and thus from (67) also

$$
\dot{c}_{\infty}\left(t_{0}\right)=0 .
$$

2) Inductive step:

We first show by induction that

$$
c_{\infty}^{(n)}= \begin{cases}\frac{1}{2} \frac{1}{t-t_{0}}\left(x^{(n)}-\frac{x^{(n-1)}}{t-t_{0}}\right) & , \text { for odd } n \\ \frac{1}{2} \frac{x^{(n)}}{t-t_{0}}, & \text { for even } n\end{cases}
$$

We have already calculated $\dot{c}_{\infty}$ (66) and $\ddot{c}_{\infty}$ (71) which are consistent with (74). Now let us assume that (74) is correct. Then it is to easy to see that for odd $n$

$$
\frac{\mathrm{d} c_{\infty}^{(n)}}{\mathrm{d} t}=\frac{\mathrm{d}}{\mathrm{d} t}\left[\frac{1}{2} \frac{1}{t-t_{0}}\left(x^{(n)}-\frac{x^{(n-1)}}{t-t_{0}}\right)\right]=\frac{1}{2} \frac{x^{(n+1)}}{t-t_{0}}=c_{\infty}^{(n+1)}
$$

and furthermore

$$
\frac{\mathrm{d} c_{\infty}^{(n+1)}}{\mathrm{d} t}=\frac{\mathrm{d}}{\mathrm{d} t}\left[\frac{1}{2} \frac{x^{(n+1)}}{t-t_{0}}\right]=\frac{1}{2} \frac{1}{t-t_{0}}\left(x^{(n+2)}-\frac{x^{(n+1)}}{t-t_{0}}\right)=c_{\infty}^{(n+2)}
$$

hence we have proven that (74) is indeed the correct term for the $n$-th derivative of (64) in the limit $\Omega_{k} \rightarrow \infty$.

For even $n$, (74) is equivalent to $x^{(n)}(t)=2 c_{\infty}^{(n)} \cdot\left(t-t_{0}\right)$ which leads to

$$
x^{(n)}\left(t_{0}\right)=0
$$

Using (77) and the rule of L'Hôpital in (74), it follows immediately

$$
c_{\infty}^{(n)}\left(t_{0}\right)=\lim _{t \rightarrow t_{0}} c_{\infty}^{(n)}(t)=0
$$

for odd $n$.

Finally from (69) it follows per induction that

$$
x^{(n)}(t)=2\left(c_{\infty}^{(n)}(t) \cdot t+n c_{\infty}^{(n-1)}(t)\right) \forall n .
$$

As $x^{(n)}\left(t_{0}\right)=0$ for even $n(77)$ and $c_{\infty}^{(n)}\left(t_{0}\right)=0$ for odd $n(78)$ we see that

$$
c_{\infty}^{(n)}\left(t_{0}\right)=0
$$


for all even $n$.

Thus we conclude that $c_{\infty}^{(n)}\left(t_{0}\right)=0$ for all $n \geq 1$.

The Taylor expansion of $\dot{c}_{\infty}(t)$ around $t_{0}$ is

$$
\dot{c}_{\infty}(t)=\sum_{n=1}^{\infty} \frac{c_{\infty}^{(n)}\left(t_{0}\right)}{n !}\left(t-t_{0}\right),
$$

and since $c^{(n)}\left(t_{0}\right)=0$ for all $\mathrm{n}$, it follows

$$
\dot{c}_{\infty}(t) \equiv 0 \quad \forall t \Rightarrow c_{\infty}=\text { const }
$$

Thus we conclude that for the observable universe the speed of light is indeed constant.

\section{Conclusions}

We have assumed a second time-like dimension with its own characteristic speed and length and the decomposability of any spacetime vector into a vector each for the first and the second time dimension. As a consequence of these assumptions, we have derived two fundamental results:

1) the existence of a smallest length scale which we have identified with the Planck length and

2) the constancy of the vacuum speed of light for the observable universe.

For very small length scales of the present universe, or for the very early universe, we have derived an expression for the speed of light. We see that for both cases, the speed of light is not constant, but depends on space and time.

This is consistent with current results from loop quantum gravity or string theory [34] [35] on the non-constancy of light speed.

Finally we here give a hint about the correctness of the assumptions of theories explaining large scale structures of the universe due to a variable speed of light in the early universe [11].

\section{Acknowledgements}

The research was partly funded by the Marie Curie Actions of the European Union's Seventh Framework Programme (FP7/2007-2013) under REA grant agreement no 609405 (COFUNDPostdocDTU).

\section{References}

[1] Maxwell, J.C. (1864) A Dynamical Theory of the Electromagnetic Field. Transactions of the Royal Society, 155, 459-512.

[2] Michelson, A.A. (1881) The Relative Motion of the Earth and the Luminiferous Ether. American Journal of Science, 22, 120-129.

https://doi.org/10.2475/ajs.s3-22.128.120

[3] Michelson, A.A. and Morely, E.W. (1887) On the Relative Motion of the Earth and the Luminiferous Ether. American Journal of Science, 34, 333-345.

https://doi.org/10.2475/ajs.s3-34.203.333

[4] Essen, L. (1955) A New Æther-Drift Experiment. Nature, 175, 793-794.

https://doi.org/10.1038/175793a0 
[5] Trimmer, W.S., et al. (1973) Experimental Search for Anisotropy in the Speed of Light. Physical Review D, 8, 3321-3326. https://doi.org/10.1103/PhysRevD.8.3321

[6] Wolf, P., et al. (2003) Tests of Lorentz Invariance using a Microwave Resonator. Physical Review Letters, 90, 060402. https://doi.org/10.1103/PhysRevLett.90.060402

[7] Hermann, S., et al. (2009) Rotating Optical Cavity Experiment Testing Lorentz Invariance at the $10^{-17}$ Level. Physical Review D, 80, 105011. https://doi.org/10.1103/PhysRevD.80.105011

[8] Nagel, M., et al. (2015) Direct Terrestrial Test of Lorentz Symmetry in Electrodynamics to $10^{-18}$. Nature Communications, 6, 8174 . https://doi.org/10.1038/ncomms9174

[9] Einstein, A. (1905) Zur Elektrodynamik bewegter Körper. [On the Electrodynamics of Moved Bodies.] Annals of Physics, 322, 891-921. https://doi.org/10.1002/andp.19053221004

[10] Amelino-Camelia, G., et al. (1998) Potential Sensitivity of Gamma-Ray Burster Observations to Wave Dispersion in Vacuo. Nature, 393, 763-765. https://doi.org/10.1038/ncomms9174

[11] Albrecht, A. and Magueijo, J. (1999) A Time Varying Speed of Light as a Solution to Cosmological Puzzles. Physical Review D, 59, Article ID: 043516. https://doi.org/10.1103/PhysRevD.59.043516

[12] Deriglazov, A.A. and Ramírez, W.G. (2015) World-Line Geometry Probed by Fast Spinning Particle. Modern Physics Letters A, 30, Article ID: 1550101. https://doi.org/10.1142/S0217732315501011

[13] Deriglazov, A.A. and Ramírez, W.G. (2016) Ultra-Relativistic Spinning Particle and a Rotating Body in External Fields. Advances in High Energy Physics, 3, Article ID: 1376016.

[14] Deriglazov, A.A. and Ramírez, W.G. (2017) Mathisson Papapetrou Tilczyjew Dixon (MPTD) Equations in Ultra-Relativistic Regime and Gravimagnetic Moment. International Journal of Modern Physics D, 26, Article ID: 1750047. https://doi.org/10.1142/S021827181750047X

[15] Wheeler, J.A. (1955) Geons. Physical Review, 97, 511-536. https://doi.org/10.1103/PhysRev.97.511

[16] Klein, O. (1956) Generalization of Einstein's Theory of Gravitation Considered from the Point of View of Quantum Field Theory. Mercier and Kervaire, 58-68, 155, 459-512.

[17] Landau, L.D., et al. (1954) The Removal of Infinities in Quantum Electrodynamics. Doklady Akademii Nauk SSSR, 95, 497.

[18] Planck, M. (1899) Über irreversible Strahlungsvorgänge. [About Irreversible Radiation.] Sitzungsberichte der Preussischen Akademie der Wissenschaften, 5, 440-481.

[19] Faizal, M. (2014) Deformation of the Wheeler? DeWitt Equation. International Journal of Modern Physics A, 29, Article ID: 1450106. https://doi.org/10.1142/S0217751X14501061

[20] Pramanik, et al. (2015) Path Integral Quantization Corresponding to the Deformed Heisenberg Algebra. Annals of Physics, 362, 24-35.

[21] Faizal, M., et al. (2016) Time Crystals from Minimum Time Uncertainty. The European Physical Journal C, 76, 30. https://doi.org/10.1140/epjc/s10052-016-3884-4

[22] Bardakçi, K., et al. (1969) Unitary Closed Loops in Reggeized Feynman Theory. Physical Review, 185, 1910-1917. https://doi.org/10.1103/PhysRev.185.1910 
[23] Nambu, Y. (1970) Quark Model and the Factorization of the Veneziano Amplitude. Symmetries and Quark Models, Gordon and Breach, 269-278.

[24] Green, M. (1987) Superstring Theory. Cambridge University Press.

[25] Lüst, D. and Theisen, S. (1989) Lectures on String Theory. Springer Verlag Berlin, Heidelberg.

[26] Witten, E. (1995) String Theory in Various Dimensions. Nuclear Physics B, 443, 85-126.

[27] Tegmark, M. (1997) On the Dimensionality of Spacetime. Classical and Quantum Gravity, 17, L69-L75. https://doi.org/10.1088/0264-9381/14/4/002

[28] Bars, I. and Kounnas, C. (1997) Theories with Two Times. Physics Letters, 402, 25-32.

[29] Bars, I. and Kounnas, C. (1997) String and Particles with Two Times. Physical Review $D$, 56, 3664. https://doi.org/10.1103/PhysRevD.56.3664

[30] Chen, X. (2008) Three Dimensional Time Theory: To Unify the Principles of Basic Quantum Physics and Relativity.

[31] Nambu, Y. (1970) Lectures at the Copenhagen Symposium.

[32] Goto, T. (1971) Relativistic Quantum Mechanics of One-Dimensional Mechanical Continuum and Subsidiary Condition of Dual Resonance Model. Progress of Theoretical Physics, 46, 1560. https://doi.org/10.1143/PTP.46.1560

[33] Collins, P.D.B., Martin, A.D. and Squires, E.J. (1989) Particle Physics and Cosmology. Wiley, New York. https://doi.org/10.1002/3527602828

[34] Kiritsis, E. (1999) Supergravity, D-Brane Probes and Thermal Super Yang-Mills: A Comparison. Journal of High Energy Physics, 9910, 010.

[35] Alexander, S. (2000) On the Varying Speed of Light in a Brane-Induced FRW Universe. Journal of High Energy Physics, 0011, 017. 